\title{
A Mesothelial Cyst Presenting as Inguinal Mass: Two Case Reports and Literature Review
}

\author{
Kyeong Woon Choi ${ }^{\mathrm{a}}$, Woo Yong Lee ${ }^{\mathrm{a}, \mathrm{b}}$
}

\begin{abstract}
The differential diagnosis of inguinal mass such as hernia, hydrocele, spermatocele, undescended testes, spermatic cord cyst, lymphangioma, lymphadenopathy, and abscess varies. Mesothelial cysts of inguinal area are extremely rare. We report the cases of a 66-yearold woman with a slowly enlarging mass in the left groin for several years, and a 58-year-old man with a 7-day history of right groin pain and swelling. Excision of cystic mass was done and the mass was pathologically confirmed mesothelial cyst which is a rare cause of inguinal mass. The literature on preoperative diagnosis of mesothelial cyst in groin is also reviewed.
\end{abstract}

Keywords: Mesothelial cyst; Inguinal mass

\section{Introduction}

The differential diagnosis for an inguinal mass can be classified into five major groups: congenital abnormalities, noncongenital hernias, vascular conditions, infectious or inflammatory processes, and neoplasms. A primary cyst of the groin is a rare lesion, which, when it occurs, can very readily be mistaken for an inguinal or femoral hernia. A few case reports describe mesothelial cyst of the groin associated with the spermatic cord or round ligament [1]. We discuss the differential points between a mesothelial cyst and inguinal hernia of the groin region.

\section{Case Reports}

\section{Case 1}

A 66-year-old female presented with a slowly enlarging mass in the left groin for 5 years without pain. A history of surgery

\footnotetext{
Manuscript submitted June 6, 2018, accepted June 18, 2018

aDepartment of Surgery, Seoul Paik Hospital, Inje University College of Medicine, Seoul, Korea

${ }^{b}$ Corresponding Author: Woo Yong Lee, Departments of Surgery, Seoul Paik Hospital, Inje University College of Medicine, 9, Mareunnae-ro, Jung-gu, Seoul 04551, Korea. Email: yongaaa5972@naver.com
}

doi: https://doi.org/10.14740/jcs350w for an advanced gastric cancer (T3N2M0, seventh, UICC) prior to the appearance of the mass was present. Physical examination revealed a non-tender tense mass with smooth surface in the left inguinal area which was not reduced by manual pressure in the supine position, nor was it changed in size by Valsalva maneuver. Also, no evidence of inflammation or incarceration was noted.

The patient appeared well-developed and had stable vital signs as well as normal laboratory results. Plain X-ray of the pelvis showed no abnormality. Computed tomography (CT) showed no recurrence of gastric cancer but larger left inguinal cystic mass $(3.6 \times 5.6 \mathrm{~cm})$ compared to the previous one $(3.0$ $\times 2.0 \mathrm{~cm}$ ) (Fig.1). Relied on CT finding, lymphangioma was strongly suspected.

At surgery, a unilocular cyst of $4.5 \times 4.5 \mathrm{~cm}$ in diameter with a smooth, glistening, and translucent surface was found to be attached to the round ligament (Fig. 2). Pathology revealed a cystic-mass-like lesion, measuring $4.5 \times 4.5 \mathrm{~cm}$ in diameter. The outer surface is covered with adipose tissue and thin membranous capsule-like structure. No evidence of infiltrative tumorous lesion is noted at the outer surface. On opening the cystic content is serosanguineous fluid with no evidence of mucinous content. The inner wall of the cyst is grayish white, thin and patchy area of hematoma formation. No solid component is noted throughout. Histopathologic examination revealed simple mesothelial cyst with chronic inflammation lined by regular mesothelial cells without atypia or mitosis (Fig. 3). The postoperative course was uneventful,

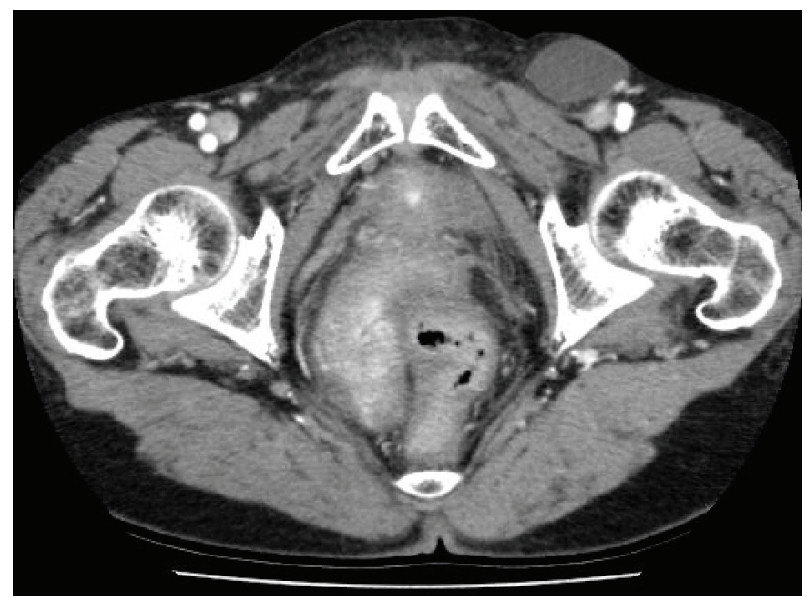

Figure 1. Computed tomography $(\mathrm{CT})$ shows a cystic mass $(3.6 \times 5.6$ $\mathrm{cm}$ ) at left inguinal region (previous size: $3.6 \times 5.1 \mathrm{~cm}$ ). 


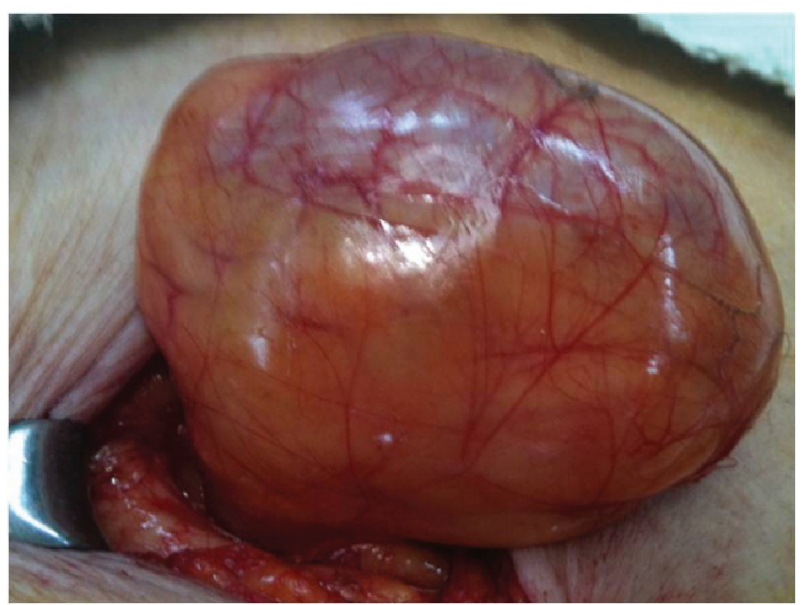

Figure 2. The extracted tumor is covered with adipose tissue, thin membranous capsule, and serous fluid collection.

and the patient was discharged from the hospital in good condition.

\section{Case 2}

A previously healthy 58-year-old man presented to the hospital with a 7-day history of right groin pain and swelling. The patient had no specific past history. Physical examination was remarkable for a large, non-tender, and reducible right groin mass in inguinal area. The testes were palpable bilaterally, and no other abnormalities. The patient appeared well-developed and had stable vital signs as well as normal laboratory results. At surgery, a unilocular cyst of $4 \times 4 \mathrm{~cm}$ in diameter with a smooth, glistening, and translucent surface was found to be attached to the superiorly and medial to the spermatic cord. The hernia sac was adherent to the medial aspect of the cyst. Pathology revealed a cystic-mass-like lesion, measuring $3 \times$ $3 \mathrm{~cm}$ in diameter and hernia sac. Histopathologic examination revealed simple mesothelial cyst with chronic inflammation lined by regular mesothelial cells without no atypia or mitosis. The postoperative course was uneventful, and the patient was discharged from the hospital in good condition.

\section{Discussion}

Inguinal mass in a woman includes hernia, enlarged lymph nodes, parasitic infection, extraperitoneal endometriosis, varix, ganglion cyst, and malignant tumors such as lymphoma, leiomyoma, etc $[1-4,5]$. A mesothelial cyst of the round ligament is relatively rare and it usually is misdiagnosed as an inguinal or femoral hernia. They are usually not considered in the differential diagnosis of groin masses and may be identified only at the time of an attempted herniorrhaphy.

The round ligament of the uterus is a derivative of gubernaculums and attaches to the paramesonephric duct near the uterotubal junction during development at 9 weeks' gestation [2]. It extends caudally through the inguinal canal to the labio-

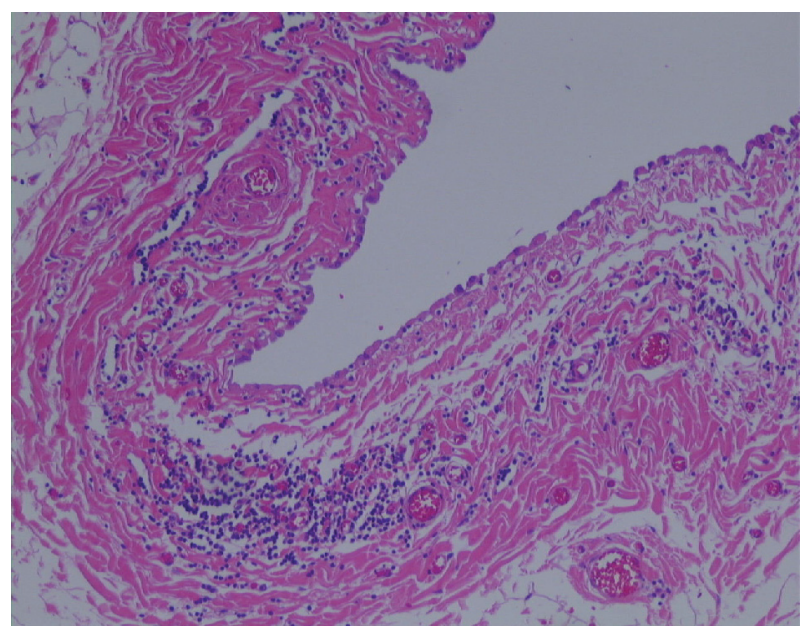

Figure 3. Cyst wall lined with a single layer of flat epithelium consisted of mesothelial origin (H\&E stain, $\times 200)$.

scrotal swelling, forming a fibrous band by the end of the first trimester of pregnancy. Nuck's canal, a portion of peritoneum known in mass as processus vaginalis, carries some layers of the abdominal wall to be incorporated in the round ligament.

There have been two theories suggested for the development of mesothelial cyst of the round ligament. The first one is a flawed obliteration of Nuck's canal. In males, a comparable flaw in the obliteration may result in a cystic hydrocele or a hydrocele of the spermatic cord. According to this theory, a round ligament cyst is the same disease as a cyst of the Nuck's canal. The other one is the inclusion of embryonic mesenchymal mesothelial elements or remnants during the development of the round ligament $[6,7]$.

Pathologic examination reveals a thin-walled, unilocular cyst that usually contains serous material. Cysts of mesothelial investment of the round ligament are lined with a single layer of flattened, cuboidal cells that have the characteristic appearance of mesothelial cells and are thin-walled, with smooth muscle and capillaries in the walls. Immunohistochemical staining showed the cell surface and wall positive of calretinin.

Clinically, a mesothelial cyst of the round ligament is usually misdiagnosed as an inguinal hernia or common benign cyst. In fact, a mesothelial cyst may be associated with an inguinal hernia in about $30-40 \%$ of cases $[2,8]$. A PubMed review of the literature produced a few case reports of mesothelial cyst in the groin (Table 1). Table 1 shows preoperative misdiagnosis and whether the hernia sac existed [2, 9-11].

Ultrasonography is the imaging modality of choice for the inguinal mass due to the easy application and availability of real-time information about the peristalsis or changes by coughing or Valsalva maneuver [12]. In addition to showing the similar pattern as in ultrasonography, multidetector CT scan more efficiently differentiates other malignant lesions. Such imaging studies may be adjunctive, diagnostic modality that is readily available in acute care settings to make diagnosis, but not in non-specific conditions. Diagnosis of mesothelial cysts is difficult and definitely needs pathologic confirmation.

Complete surgical excision is the mainstay of therapy. Symptomatic or growing cysts of the groin lesion should be 
Table 1. : Literature Data of Mesothelial Cyst in Groin

\begin{tabular}{lllllll}
\hline Authors (year) & Sex/age & Misdiagnosis & Location & Size(cm) & Reducibility & Hernia sac \\
\hline Aarabi et al (2010) [9] & M/10 & Inguinal mass & Spermatic cord & $6.5 \times 1.8$ & Yes & Yes \\
Kim et al (2010) [10] & F/76 & Mimicking a metastasis & Round ligament & $21 \times 10$ & No & No \\
Vaos et al (2009) [11] & M/2 & Undescended testis & Spermatic cord & $1.5 \times 1.0$ & No & No \\
Ryley et al (2004) [2] & F/31 & Inguinal hernia & Round ligament & $4.8 \times 2.3$ & No & N0 \\
\hline
\end{tabular}

M: male; F: female.

excised and the inguinal canal should be explored so that inguinal hernia should be ruled out.

\section{Conclusions}

In conclusion, although rare, a mesothelial cyst of the round ligament should be considered in differentiating inguinal masses for diagnosis.

\section{Conflicts of Interest}

There was no conflict of interest from all authors in this case report.

\section{References}

1. Harper GB, Jr., Awbrey BJ, Thomas CG, Jr., Askin FB. Mesothelial cysts of the round ligament simulating inguinal hernia. Report of four cases and a review of the literature. Am J Surg. 1986;151(4):515-517.

2. Ryley DA, Moorman DW, Hecht JL, Alper MM. A mesothelial cyst of the round ligament presenting as an inguinal hernia after gonadotropin stimulation for in vitro fertilization. Fertil Steril. 2004;82(4):944-946.

3. Rhee CS, Kim JS, Woo SK, Suh SJ. MRI of round ligament leiomyoma associated with Mayer-Rokitansky-Kuster-
Hauser syndrome. Abdom Imaging. 1999;24(2):202-204.

4. Cheng D, Lam H, Lam C. Round ligament varices in pregnancy mimicking inguinal hernia: an ultrasound diagnosis. Ultrasound Obstet Gynecol. 1997;9(3):198-199.

5. Warshauer DM, Mandel SR. Leiomyoma of the extraperitoneal round ligament: CT demonstration. Clin Imaging. 1999;23(6):375-376.

6. Anderson CC, Broadie TA, Mackey JE, Kopecky KK. Hydrocele of the canal of Nuck: ultrasound appearance. Am Surg. 1995;61(11):959-961.

7. Stickel WH, Manner M. Female hydrocele (cyst of the canal of Nuck): sonographic appearance of a rare and littleknown disorder. J Ultrasound Med. 2004;23(3):429-432.

8. Khanna PC, Ponsky T, Zagol B, Lukish JR, Markle BM. Sonographic appearance of canal of Nuck hydrocele. Pediatr Radiol. 2007;37(6):603-606.

9. Aarabi S, Drugas G, Avansino JR. Mesothelial cyst presenting as an irreducible inguinal mass. J Pediatr Surg. 2010;45(6):e19-21.

10. Kim BM, Lee JY, Han YH, Kim SY, Seo JW, Kim YH, Cha SJ, et al. Mesothelial cyst of the round ligament mimicking a metastasis: a case report. Korean J Radiol. 2010;11(3):364-367.

11. Vaos G, Zavras N, Velaoras K, Ereikat K. Mesothelial cyst of the spermatic cord as a cause of acquired cryptochidism. Hernia. 2009;13:439-441.

12. Oh SN, Jung SE, Lee JM, Chung JH, Park GS. Sonographic diagnosis of a round ligament cyst in the inguinal area. J Clin Ultrasound. 2007;35(4):226-228. 